\title{
A Feeble Window on Leptophilic Dark Matter
}

\author{
Sam Junius ${ }^{* a, b, c}$, Laura Lopez-Honorez ${ }^{a, b}$ and Alberto Mariotti ${ }^{b, c}$ \\ a Service de Physique Théorique, Université Libre de Bruxelles, C.P. 225, B-1050 Brussels, \\ Belgium \\ ${ }^{b}$ Theoretische Natuurkunde \& The International Solvay Institutes, Vrije Universiteit Brussel, \\ Pleinlaan 2, B-1050 Brussels, Belgium \\ ${ }^{c}$ Inter-University Institute for High Energies, Vrije Universiteit Brussel, Pleinlaan 2, B-1050 \\ Brussels, Belgium \\ E-mail: sam.junius@vub.be,llopezho@ulb.ac.be,alberto.mariotti@vub.be
}

\begin{abstract}
We study a leptophilic dark matter scenario involving feeble dark matter coupling to the Standard Model (SM) and compressed dark matter-mediator mass spectrum. In particular, we consider a simplified model where the SM is extended with one Majorana fermion, the dark matter, and one charged scalar, the mediator, coupling to the SM leptons through a Yukawa interaction. We first discuss the dependence of the dark matter relic abundance on the Yukawa coupling going continuously from the well known freeze-out paradigm to a novel production mechanism called conversion driven freeze-out. Focusing on the latter, we then exploit the macroscopic decay length of the charged scalar to study the resulting long-lived-particle signatures at collider and to explore the experimental reach on the viable portion of the parameter space.
\end{abstract}

European Physical Society Conference on High Energy Physics - EPS-HEP2019 -

10-17 July, 2019

Ghent, Belgium

${ }^{*}$ Speaker. 


\section{Introduction}

As of today, the most conventional paradigm for dark matter (DM) has been the so-called Weakly Interacting Massive Particle (WIMP). In the WIMP scenario, the DM is produced in the early Universe through the freeze-out mechanism, leading typically to the correct DM relic abundance for electroweak size couplings and masses. This is however not the only possibility to obtain the right DM abundance. By varying the DM mass, its coupling strength to the Standard Model (SM) and/or within the dark sector, one can generate DM through different mechanisms during the cosmological evolution of the Universe, see e.g. [1, 2,3]. In some parts of this parameter space, the DM happens to be very feebly coupled to the SM, i.e. with couplings much more suppressed than for the WIMP case.

We will study in details the mechanism of dark matter production in the early universe, focusing mainly on the cases when the interactions are feeble. In particular, we will highlight the main features of an intermediate stage of DM coannihilation freeze-out happening out of chemical equilibrium (CE) with the SM plasma, also called conversion driven freeze-out. Such a scenario has already been pointed out in [3] and mainly studied for dark matter coupling to quarks [3, 4]. Here instead we focus on the case of a leptophilic dark matter model. Conversion processes between the mediator and the dark matter will play a central role in defining the evolution of the DM abundance and they will have to be taken into account in the study of the DM/mediator Boltzmann equations.

The feeble coupling of the DM to the mediator that will be considered, allows for a macroscopic decay length of the mediator that can be observed at colliders through e.g. charged and/or disappearing tracks. Recently, searches have been devoted to such long lived particle signatures arising in DM models, that we will study here (see also [5] for more details). Notice that due to the feeble coupling involved, direct and indirect detection dark matter searches are challenging. Unconventional signatures at the LHC can hence provide the main experimental probes for the class of models studied here.

In Sec. 2, we will introduce a simplified leptophilic DM model serving as an illustrative case to discuss the early Universe dark matter production in Sec. 3 and collider prospects in Sec. 4. Finally, we will conclude in Sec. 5.

\section{The Model}

We work in a minimal extension of the Standard Model (SM) involving a Majorana fermion $\chi$ dark matter coupled to SM leptons through the exchange of charged scalar mediator $\phi$. The Lagrangian encapsulating the BSM physics reads

$$
\mathscr{L} \supset \frac{1}{2} \bar{\chi} \gamma^{\mu} \partial_{\mu} \chi-\frac{m_{\chi}}{2} \bar{\chi} \chi+\left(D_{\mu} \phi\right)^{\dagger} D^{\mu} \phi-m_{\phi}^{2}|\phi|^{2}-\lambda_{\chi} \phi \bar{\chi} l_{R}-\lambda_{H} H^{\dagger} H \phi^{\dagger} \phi+\text { h.c. }
$$

where $m_{\chi}$ is the dark matter mass, $m_{\phi}$ is the mediator mass, $\lambda_{\chi}$ denotes the Yukawa coupling between the dark matter, the right handed SM lepton $l_{R}$ and the mediator and $\lambda_{H}$ denotes the quartic coupling of the mediator to the SM Higgs, which we set to 0.1 as an illustrative case. We have assumed that a $Z_{2}$ symmetry prevents the dark matter to decay directly to SM particles. Both $\chi$ and $\phi$ are odd under the $Z_{2}$ symmetry while the SM fields are even and we assume $m_{\phi}>m_{\chi}$. 
Here, we study DM coupling to leptons only, i.e. $l_{R}=e_{R}, \mu_{R}, \tau_{R}$, and present all our results for the $l_{R}=\mu_{R}$ case. The case of a DM feebly coupled to quarks has been studied in a very similar model in $[3,4,6,7]$.

Considering the minimal SM extension of Eq. (2.1), there are three free parameters in our model, the dark matter mass $m_{\chi}$, the mediator mass $m_{\phi}$ and the Yukawa coupling $\lambda_{\chi}$. Here in particular, we exchange the mediator mass for the mass splitting $\Delta m=m_{\phi}-m_{\chi}$ to obtain a minimal set of free parameters of the model we will consider.

\section{Dark Matter Abundance}

In order to compute the number density evolution of a set of species in kinetic equilibrium, one has to solve a coupled set of Boltzmann equations taking the form:

$$
H x s \frac{d Y_{i}}{d x}=-\sum_{j k} \gamma_{i j \rightarrow k l}\left(\frac{Y_{i} Y_{j}}{Y_{i}^{e q} Y_{j}^{e q}}-\frac{Y_{k} Y_{l}}{Y_{k}^{e q} Y_{l}^{e q}}\right)-\sum_{j k} \gamma_{i j \rightarrow k}\left(\frac{Y_{i} Y_{j}}{Y_{i}^{e q} Y_{j}^{e q}}-\frac{Y_{k}}{Y_{k}^{e q}}\right)
$$

where $Y_{i}=n_{i} / s$ is the comoving number density of the species $i, s$ is the entropy density, the $e q$ superscript refers to equilibrium, $x=m_{\chi} / T$ with $T$ the thermal bath temperature, and $H=H(x)$ is the Hubble rate at time $x$. Here we have considered contributions from 4 and 3 particle interactions inducing the $\gamma_{i j \rightarrow k l}$ and $\gamma_{i j \rightarrow k}$ reaction densities. There is a direct correspondence between these reaction densities and the thermal averaged scattering cross sections/decay rates going as follows:

$$
\begin{aligned}
\gamma_{i j \rightarrow k l} & =\iint_{e} d \phi_{i} d \phi_{j} f_{i}^{e q} f_{j}^{e q} \iint d \phi_{k} d \phi_{l}(2 \pi)^{4} \delta^{4}\left(p_{i}+p_{j}-p_{k}-p_{l}\right)\left|\mathscr{M}_{i j \rightarrow k l}\right|^{2} \\
& =n_{i}^{e q} n_{j}^{e q}\left\langle\sigma_{i j \rightarrow k l} v_{i j}\right\rangle \\
\gamma_{i j \rightarrow k} & =\iint d \phi_{i} d \phi_{j} \int d \phi_{k} f_{k}^{e q}(2 \pi)^{4} \delta^{4}\left(p_{i}+p_{j}-p_{k}\right)\left|\mathscr{M}_{k \rightarrow i j}\right|^{2}=n_{k}^{e q} \Gamma_{k \rightarrow i j} \frac{K_{1}(x)}{K_{2}(x)}
\end{aligned}
$$

where $K_{1,2}$ denote the Bessel functions, $|\mathscr{M}|^{2}$ are the squared scattering amplitudes summed (not averaged) over initial and final degrees of freedom and $d \phi_{i}=d^{3} p_{i} /\left(2 E_{i}(2 \pi)^{3}\right)$. We neglect quantum statistical effects and we use the Maxwell Boltzmann equilibrium distributions $f_{i}^{e q}$. In the dark matter model considered here, the charged mediator $\phi$ is always in CE with the SM thermal plasma at early times because of gauge interactions. In contrast, in e.g. the context of conversion driven freeze-out, suppressed DM-mediator conversion processes may prevent chemical equilibrium (CE) between $\chi$ and $\phi$. Therefore, the following Boltzmann system has then to be solved (see e.g. [3]):

$$
\begin{aligned}
\frac{d Y_{\chi}}{d x}=\frac{-2}{H x s} & {\left[\gamma_{\chi \chi}\left(\frac{Y_{\chi}^{2}}{Y_{\chi, e q}^{2}}-1\right)+\gamma_{\chi \phi}\left(\frac{Y_{\chi} Y_{\phi}}{Y_{\chi, e q} Y_{\phi, e q}}-1\right)\right.} \\
& \left.+\gamma_{\chi \rightarrow \phi}\left(\frac{Y_{\chi}}{Y_{\chi, e q}}-\frac{Y_{\phi}}{Y_{\phi, e q}}\right)+\gamma_{\chi \chi \rightarrow \phi \phi^{\dagger}}\left(\frac{Y_{\chi}^{2}}{Y_{\chi, e q}^{2}}-\frac{Y_{\phi}^{2}}{Y_{\phi, e q}^{2}}\right)\right], \\
\frac{d Y_{\phi}}{d x}=\frac{-2}{H x s} & {\left[\gamma_{\phi \phi^{\dagger}}\left(\frac{Y_{\phi}^{2}}{Y_{\phi, e q}^{2}}-1\right)+\gamma_{\chi \phi}\left(\frac{Y_{\chi} Y_{\phi}}{Y_{\chi, e q} Y_{\phi, e q}}-1\right)\right.} \\
& \left.-\gamma_{\chi \rightarrow \phi}\left(\frac{Y_{\chi}}{Y_{\chi, e q}}-\frac{Y_{\phi}}{Y_{\phi, e q}}\right)-\gamma_{\chi \chi \rightarrow \phi \phi^{\dagger}}\left(\frac{Y_{\chi}^{2}}{Y_{\chi, e q}^{2}}-\frac{Y_{\phi}^{2}}{Y_{\phi, e q}^{2}}\right)\right],
\end{aligned}
$$




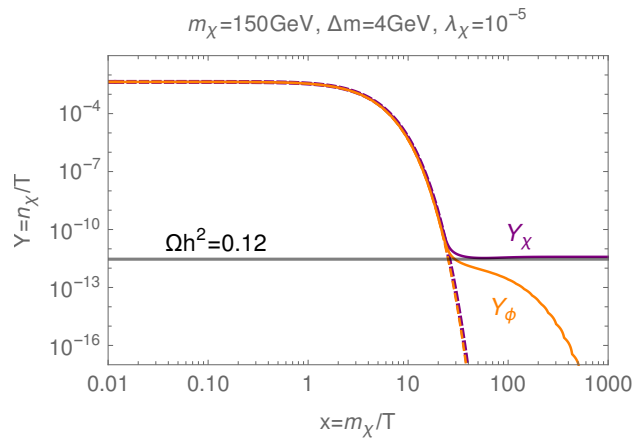

(a)

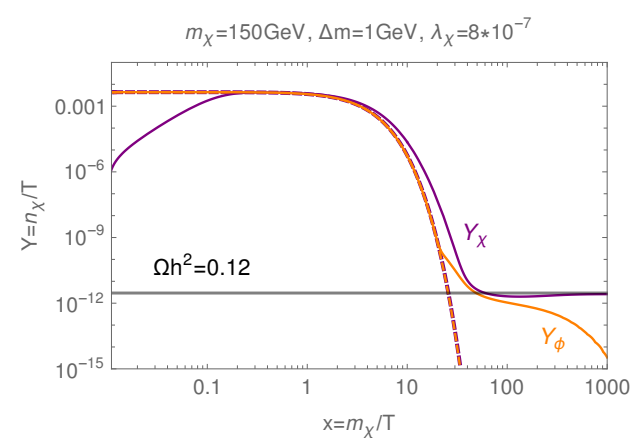

(b)

Figure 1: Evolution of the DM (purple) and mediator (orange) yield $Y$ as a function of the inverse temperature $x$ for $m_{\chi}=150 \mathrm{GeV}$. The orange and purple dashed lines denote respectively the DM and mediator equilibrium yield. LEFT: $\Delta m=4 \mathrm{GeV}$ is chosen in a way that mediator driven freeze-out reproduces the correct relic abundance for $\lambda_{\chi}=10^{-5}$. RIGHT: $\Delta m=1 \mathrm{GeV}$ such that conversion driven freeze-out reproduces the correct relic abundance for $\lambda_{\chi}=8 \times 10^{-7}$.

where $\gamma_{i j}=\gamma_{i j \rightarrow \alpha \beta}$, with $\alpha, \beta$ some SM particles in equilibrium with the bath, $\gamma_{\chi \rightarrow \phi}$ includes all conversion processes, i.e. both decays and scatterings $\gamma_{\chi \rightarrow \phi}=\left(\gamma_{\chi \alpha \rightarrow \phi \beta}+\gamma_{\chi \alpha \rightarrow \phi}\right), Y_{\phi}$ is the summed contribution of both the mediator and its antiparticle and we will use $x=m_{\chi} / T$.

In order to study the dependence of the DM relic abundance on the parameters of the model under study, we will numerically solve eq. (3.4) and (3.5) and establish different possible production mechanisms that could give rise to the correct relic density $\Omega h^{2}=0.12$ [8]. First, let us assume that the Yukawa coupling constant $\lambda_{\chi}$ is relatively large $\left(\lambda_{\chi}>\mathscr{O}\left(10^{-5}\right)\right)$. In this scenario, DM will be produced through the well studied freeze-out mechanism where initially, the processes that keep the DM in contact with the SM bath are efficient (i.e. $\gamma>H n_{\chi}^{e q}$ ) such that the DM particle is in equilibrium with the SM bath. We can subdivide the freeze-out regime into two scenarios. Firstly, DM can be kept in equilibrium (until it undergoes freeze-out, which in this case we will refer to as DM freeze-out) through its annihilation processes $\chi \chi \rightarrow S M S M$ when $\lambda_{\chi} \sim \mathscr{O}(0.1-1)$. These values of the coupling are necessary since the cross section of the processes keeping DM directly in equilibrium with the SM highly depends on the value of this constant $\left(\sigma_{a n n} \sim \lambda_{\chi}^{4}\right)$. Therefore, for smaller values of $\lambda_{\chi}$, these annihilation processes are inefficient throughout the whole evolution of the Universe. In this case, DM can be kept in equilibrium though its mediator $\phi$ via conversion processes $\chi S M \rightarrow \phi S M$. These processes keep $\chi$ in equilibrium with $\phi$, while $\phi$ itself is in equilibrium with the SM bath through gauge interactions until it freezes out itself. This makes that $\chi$ and $\phi$ will freeze-out at the same time, as can be seen in Fig. 1(a). We will dub this scenario as mediator driven freeze-out regime.

Just as the DM annihilation processes depend on $\lambda_{\chi}$, so do also the conversion processes, although less heavily $\left(\sigma_{\text {conv }} \sim \lambda_{\chi}^{2}\right)$. For $\lambda_{\chi} \sim \mathscr{O}\left(10^{-7}-10^{-6}\right)$, the conversion processes will be on the edge of being efficient, i.e. $\gamma_{\chi \rightarrow \phi} \approx H n_{\chi}^{e q}$ such that DM will not be produced through freeze-out (including mediator driven freeze-out) but through a novel production mechanism called conversion driven freeze-out. Here, $\chi$ will not be able to reach complete equilibrium with the mediator as can be seen by studying the evolution of the DM yield $Y_{\chi}(x)$ in Fig. 1(b). The DM abundance will 


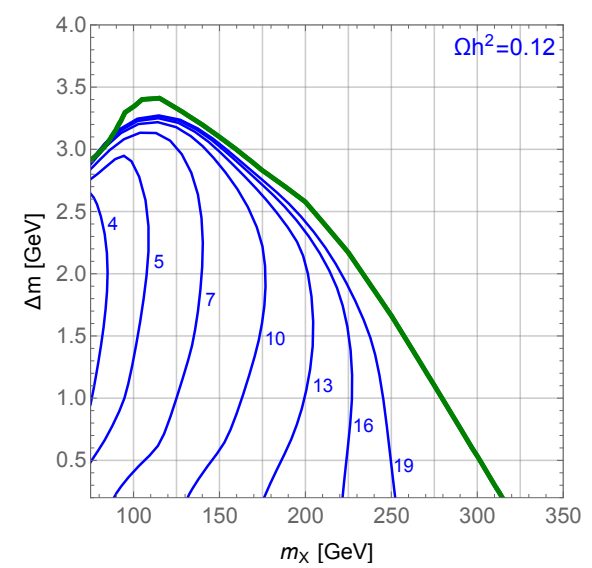

Figure 2: Viable parameter space for DM abundance through DM freeze-out (above the green line), mediator driven freeze-out (on the green line) and conversion driven freeze-out (below the green line). Contours denoting $\Omega h^{2}=0.12$ obtain through conversion driven freeze-out for fixed value of the Yukawa coupling $\lambda_{\chi} / 10^{-7}$ are shown with blue lines.

initially rise after which, due to the fact that the conversion processes are just efficient enough, it will start to convert back to the mediator however in a less efficient way as would happen during mediator driven freeze-out causing the relic density to be larger. Therefore, if mediator driven freeze-out cannot account for enough DM for a specific set of parameters, we can obtain the correct relic abundance by lowering the value of the coupling constant $\lambda_{\chi}$ and producing DM through conversion driven freeze-out to obtain the observed amount of DM in our Universe today.

Fig. 2 denotes in which region of parameter space the three previously discussed DM production mechanisms can reproduce the correct relic abundance. The green line denotes for which values of $m_{\chi}$ and $\Delta m$, mediator driven freeze-out can account for all dark matter. This can be done for many different values of $\lambda_{\chi}$, ranging from about $10^{-6}$ to approximately $10^{-2}$. For values of $m_{\chi}$ and $\Delta m$ above the green line, DM freeze-out will reproduce the correct relic abundance, while for all values below the line, conversion driven freeze-out will do this. In the latter case, the value of $\lambda_{\chi}$ has to be of the order of $10^{-7}-10^{-6}$ as denoted by the blue contours. This will give interesting signatures at collider experiments, as will be discussed in the following section.

\section{Distinct Signatures at the LHC}

The small Yukawa couplings necessary to reproduce the correct DM relic abundance through conversion driven freeze-out implies a small decay width of the charged mediator through the process $\phi \rightarrow \chi l$. For $\Delta m \ll m_{\chi}$, the decay rate for $\phi \rightarrow \chi l$ reduces at first orders in $\Delta m$ to:

$$
\Gamma_{\phi} \approx \frac{\lambda_{\chi}^{2} \Delta m^{2}}{4 \pi m_{\chi}}\left[1-\frac{2 \Delta m}{m_{\chi}}\right] \sim \frac{1}{25 \mathrm{~cm}}\left(\frac{\lambda_{\chi}}{10^{-6}}\right)^{2}\left(\frac{\Delta m}{1 \mathrm{GeV}}\right)^{2}\left(\frac{100 \mathrm{GeV}}{m_{\chi}}\right)
$$

when neglecting the lepton mass $\left(m_{l} \ll m_{\chi}, m_{\phi}\right)$. The testable signatures at colliders for this class of model is hence the pair production of charged mediators through gauge interaction and possibly their subsequent macroscopic decay into DM plus leptons. Depending on the lifetime of the mediator, three different signatures can be observed at colliders. Firstly, the lifetime of the mediator can 


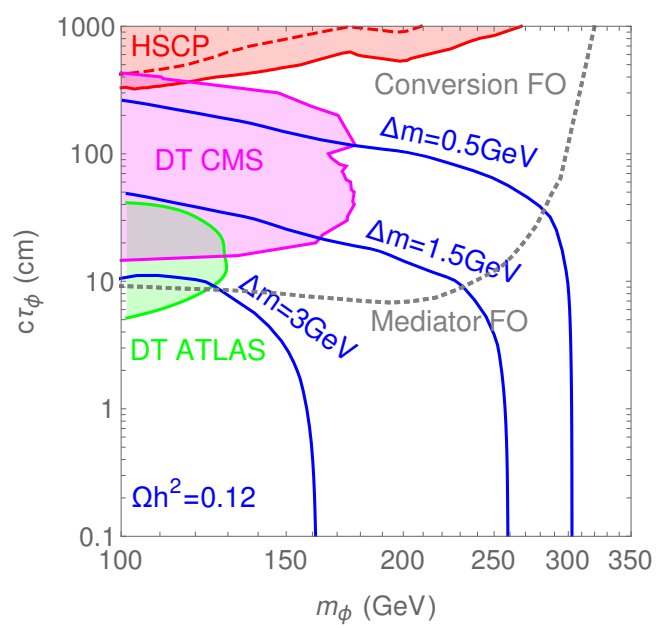

Figure 3: Proper life time of the mediator as a function of the dark matter mass. The blue contours reproduce the correct relic abundance in the $\left(m_{\chi}, c \tau_{\phi}\right)$ plane for different values of the mass-splitting $\Delta m$. The gray dotted line separates the conversion driven (top) from the mediator driven (bottom) freeze-out regime. The excluded regions resulting from heavy stable particle searches (HSCP, red region, solid (dotted) line denotes constraints from 13 (8) $\mathrm{TeV}$ searches) and disappearing tracks searches (DT, green (ATLAS) and pink (CMS) region) are also shown.

be long enough in order to escape the detector completely, leaving a charged track in the tracker. When the decay happens inside the detector, the track will stop and is referred to as a disappearing track. Finally, if the decay products of the mediator (apart from the DM) can be reconstructed, a pair of displaced leptons can be observed.

CMS $[9,10,11,12,13]$ and ATLAS [14] have put constraints on long lived particle signatures. Their projection in the $m_{\chi}$-c $\tau$ plane in our model is shown in Fig. 3, together with contours where the correct relic DM abundance is reproduced by mediator or conversion driven freeze-out for fixed values of the mass splitting $\Delta m$. While we see that searches for charged and disappearing tracks put bounds on our model, the displaced lepton searches do not. This is due to the small mass splitting that is required to reproduce the correct relic DM density making the leptons originating from the decay of the mediator very soft and hard to reconstruct.

\section{Conclusion}

We have described the effect of varying the parameters of a simplified leptophilic DM model on the way of producing dark matter. In particular, we discussed in detail the differences between DM freeze-out, mediator driven freeze-out and conversion driven freeze-out by taking into account explicitly the mediator abundance. The latter production mechanism has only been pointed out recently and can reproduce the correct relic abundance for small values of the Yukawa coupling and a compressed mass spectrum. We have also pinpointed the viable parameter space in order to account for all the DM in the three different production regimes.

Finally we have addressed the collider constraints on the model under study. Interestingly the feeble coupling involved gives rise to a long lived mediator that can a priori be tested through 
existing searches for heavy stable charged particles, disappearing charged tracks and, possibly, displaced leptons. We have recasted existing searches and projected the results within our scenario as shown in Fig. 3. As can be seen, only disappearing charged tracks and heavy stable charged particle searches provide relevant constraints on the parameter space. Displaced leptons can not help to further test our DM scenario due to the compressed mass spectrum. At this point, a large part of the parameter space is left unconstrained.

\section{Acknowledgments}

SJ is supported by a ULB PhD grant and LLH is a Research associate of the FRS-FNRS. This work is supported by the FNRS research grant F.4520.19; by the Strategic Research Program HEP and the Research Council of the Vrije Universiteit Brussel; and by the EOS - be.h project n.30820817.

\section{References}

[1] L. J. Hall, K. Jedamzik, J. March-Russell and S. M. West, Freeze-In Production of FIMP Dark Matter, JHEP 03 (2010) 080 [0911.1120].

[2] X. Chu, T. Hambye and M. H. G. Tytgat, The Four Basic Ways of Creating Dark Matter Through a Portal, JCAP 1205 (2012) 034 [1112.0493].

[3] M. Garny, J. Heisig, B. Lulf and S. Vogl, Coannihilation without chemical equilibrium, Phys. Rev. D96 (2017) 103521 [1705.09292].

[4] M. Garny, J. Heisig, M. Hufnagel and B. Lulf, Top-philic dark matter within and beyond the WIMP paradigm, Phys. Rev. D97 (2018) 075002 [1802.00814].

[5] S. Junius, L. Lopez-Honorez and A. Mariotti, A feeble window on leptophilic dark matter, JHEP 07 (2019) 136 [1904.07513].

[6] M. Garny and J. Heisig, Interplay of super-WIMP and freeze-in production of dark matter, Phys. Rev. D98 (2018) 095031 [1809.10135].

[7] G. Belanger et al., LHC-friendly minimal freeze-in models, JHEP 02 (2019) 186 [1811. 05478 ].

[8] PLAnCK collaboration, Planck 2015 results. XIII. Cosmological parameters, Astron. Astrophys. 594 (2016) A13 [1502.01589].

[9] CMS collaboration, Constraints on the pMSSM, AMSB model and on other models from the search for long-lived charged particles in proton-proton collisions at sqrt(s) $=8 \mathrm{TeV}$, Eur. Phys. J. C75 (2015) 325 [1502.02522].

[10] CMS collaboration, Search for heavy stable charged particles with $12.9 \mathrm{fb}^{-1}$ of 2016 data, .

[11] CMS collaboration, Search for disappearing tracks as a signature of new long-lived particles in proton-proton collisions at $\sqrt{s}=13 \mathrm{TeV}, 1804.07321$.

[12] CMS collaboration, Search for Displaced Supersymmetry in events with an electron and a muon with large impact parameters, Phys. Rev. Lett. 114 (2015) 061801 [1409.4789].

[13] CMS collaboration, Search for displaced leptons in the e-mu channel, CMS-PAS-EXO-16-022.

[14] ATLAS collaboration, Search for long-lived charginos based on a disappearing-track signature in pp collisions at $\sqrt{s}=13 \mathrm{TeV}$ with the ATLAS detector, JHEP 06 (2018) 022 [1712.02118]. 